\title{
Intestinal helminthiases and schistosomiasis among school children in an urban center and some rural communities in southwest Nigeria
}

\author{
Olufemi Moses AGBOLADE ${ }^{1) *}$, Ndubuisi Chinweike AGU ${ }^{1)}$, Oluseyi Olusegun ADESANYA ${ }^{2)}$, \\ Adedayo Olugbenga ODEJAYI ${ }^{1}$, Aliu Adekunle ADIGUN ${ }^{1}$, Emmanuel Babatunde ADESANLU1), \\ Flourish George OGUNLEYE ${ }^{1)}$, Adetoun Omolayo SODIMU1), Stella Ajoke ADESHINA ${ }^{2)}$, \\ Ganiyat Olusola BISIRIYU2), Oluwatosin Ibiyemi OMOTOSO ${ }^{2)}$ and Karen Mfon UDIA ${ }^{2)}$ \\ ${ }^{1)}$ Parasitology and Medical Entomology Laboratory, Department of Plant Science and Applied Zoology, \\ Olabisi Onabanjo University, P.M.B. 2002, Ago-Iwoye, Ogun State, \\ ${ }^{2)}$ Department of Microbiology, Olabisi Onabanjo University, P.M.B. 2002, Ago-Iwoye, Ogun State, Nigeria
}

\begin{abstract}
Intestinal helminths and schistosomiasis among school children were investigated in an urban and some rural communities of Ogun State, southwest Nigeria. Fecal samples of 1,059 subjects (524 males, 535 females) aged 3-18 years were examined using direct smear and brine concentration methods between June 2005 and November 2006. The pooled prevalence of infection was $66.2 \%$. Ascaris lumbricoides showed the highest prevalence (53.4\%) $(P$ $<0.001)$ followed by hookworms $(17.8 \%)$, Trichuris trichiura $(10.4 \%)$, Taenia sp. $(9.6 \%)$, Schistosoma mansoni $(2.3 \%)$, Strongyloides stercoralis $(0.7 \%)$, Schistosoma haematobium $(0.6 \%)$, and Enterobius vermicularis $(0.3 \%)$. The prevalences of $A$. lumbricoides, hookworms, Taenia sp., S. mansoni, and S. stercoralis in the urban centre were simi$\operatorname{lar}(P>0.05)$ to those in the rural communities. The fertile and infertile egg ratios of $A$. lumbricoides in the urban centre and the rural communities were 13: 1 and 3.7: 1, respectively. Each helminth had similar prevalences among both genders $(P>0.05)$. The prevalence of $A$. lumbricoides increased significantly with age $(P<0.001)$. The commonest double infections were Ascaris and hookworms, while the commonest triple infections were Ascaris, hookworms, and Trichuris. The study demonstrates the need for urgent intervention programmes against intestinal helminthiases and schistosomiasis in the study area.
\end{abstract}

Key words: Ascaris lumbricoides, Trichuris trichiura, Strongyloides stercoralis, Taenia sp., Schistosoma mansoni, Schistosoma haematobium, prevalence, southwest Nigeria

Intestinal helminthiases and schistosomiasis have long been recognized as human infections of significant public health importance especially in the tropics and are known to be usually more common in chil-

\footnotetext{
- Received 31 May, accepted after revision 10 August 2007.

*Corresponding author (e-mail: agbolmos@yahoo.com, agbolmos@hotmail.com)
}

dren than in adults (Nokes et al., 1991; WHO, 1993; Gillespie, 2001). Although relatively easy to control, several reports have shown that these human infections have remained endemic in many parts of the tropical world (Utzinger et al., 2002; Clennon et al., 2004; Poggensee et al., 2005).

Recent reports on intestinal helminthiases and schistosomiasis from Nigeria include those of Anosike 
et al. (2001), Bassey and Umar (2004), Ahmed et al. (2004), and Idowu and Rowland (2006). To the best of our knowledge, there is none on the epidemiology of intestinal helminthiasis from Ijebu-Ode Local Government Area (ILGA) and Odogbolu Local Government Area (OLGA) of Ogun State, southwest Nigeria. Moreover, the only previous report on urinary schistosomiasis involving school children in the 2 Local Government Areas was based exclusively on questionnaires (Ekpo and Mafiana, 2004).

The present study aimed to figure out the epidemiological picture of intestinal helminthiases and schistosomiasis among school children in some communities of OLGA and ILGA of Ogun State, southwest Nigeria. It is hoped that the findings of this study will assist the health planners and workers in the study area in their bid to provide health for all.

The study area consisted of Okun-Owa and IjeshaIjebu in OLGA, and Ijebu-Ode, Iloti, Odonoko, Irawo, Isiwo, and Odolewu in ILGA. Both Local Government Areas are in Ijebu division of Ogun State, Nigeria. The study area lies in the rainforest belt within latitudes $6^{\circ}$ $30^{\prime}$ and $6^{\circ} 40^{\prime} \mathrm{N}$, longitudes $3^{\circ} 40^{\prime}$ and $3^{\circ} 50^{\prime} \mathrm{E}$ (Fig. 1). Okun-Owa and Ijesha-Ijebu are developing rural communities whose inhabitants are predominantly Yorubas (Ijebu dialect), who engage mainly in subsistence farming. Okun-Owa has 3 public schools, 1 public secondary school, while Ijesha-Ijebu has 1 public primary school. Each of the communities has 1 public primary health centre and a private clinic.

Ijebu-Ode is both urban and cosmopolitan, it is the headquarters of ILGA and its population consists of civil servants, artisans, traders and farmers. The town has 1 secondary health centre, many public and private health centers and about 30 public primary schools. Iloti, Odonoko, Irawo, Isiwo, and Odolewu are rural communities in ILGA. Each of the communities has a public primary school and a primary health centre. Many of the inhabitants are farmers.

In all the communities included in this study, potable water supply is infrequent, therefore, the inhabitants usually depend on streams (particularly in the rural communities), wells, bore-holes, harvested rain water (in rainy season) and mobile water tankers

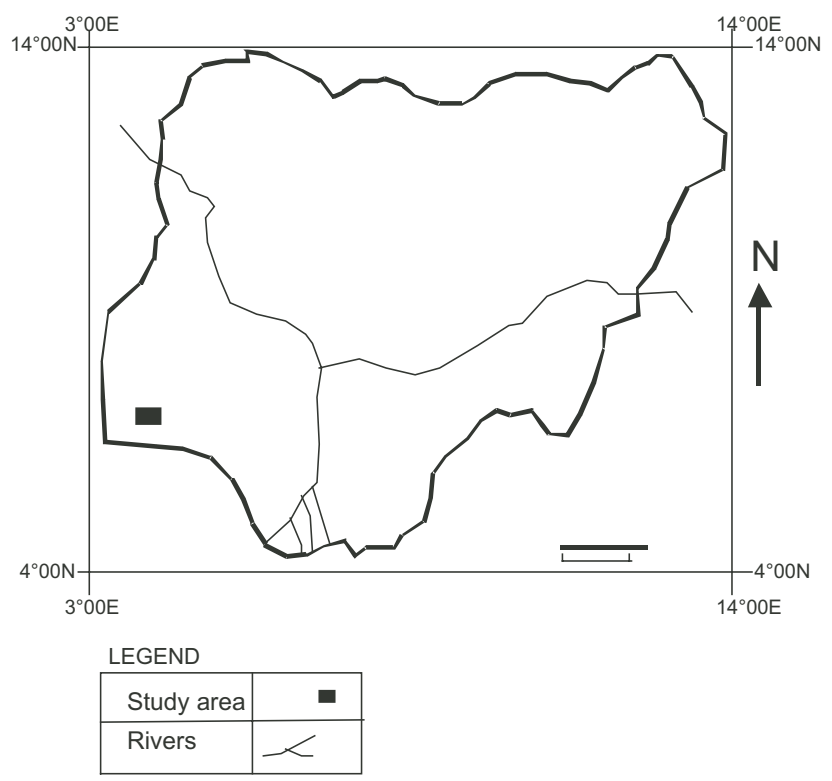

Fig. 1. Map showing the study area of intestinal helminthiases and schistosomiasis in southwest Nigeria.

for their domestic water needs. Fecal disposal facilities used by the inhabitants of all the communities include water closet, pit latrines (commonest, especially in the rural areas) and open defecation.

Prior to the commencement of the study, permission was obtained from the local education authority of each of OLGA and ILGA. The head teacher and teachers of each selected school were contacted, and the parents and pupils were adequately enlightened on the purpose of the study. All the public schools in Okun-Owa, the public primary school in Ijesha-Ijebu, 8 randomly-selected public primary schools in IjebuOde, and the public primary school in each of Iloti, Odonoko, Irawo, Isiwo, and Odolewu were included in the study. The examined population size was 1,059 (524 males, 535 females) and the age range was 3-18 years. A total of 662 and 397 subjects were examined in the rural communities and the urban center, respectively. The study was performed between June 2005 and November 2006.

For each participating school child, a questionnaire was administered to obtain information, such as age, sex, source of water for drinking, and other purposes, toilet facilities, and hygiene practices. Labeled sample bottles were distributed to the school children for a lit- 
Table 1. Prevalence of parasitic helminths in the urban center and rural communities surveyed in southwest Nigeria

\begin{tabular}{|c|c|c|}
\hline \multirow[t]{2}{*}{ Helminths } & \multicolumn{2}{|c|}{ No. (\%) egg positive cases } \\
\hline & Urban center & Rural communities \\
\hline No. of cases examined & 397 & 662 \\
\hline Ascaris lumbricoides & $212(53.4)$ & $353(53.3)$ \\
\hline Hookworms & $71(17.9)$ & $118(17.8)$ \\
\hline Trichuris trichiura & $8(2.0)$ & $102(15.4)$ \\
\hline Taenia sp. & $36(9.1)$ & $66(10.0)$ \\
\hline Schistosoma mansoni & 11 (2.8) & $13(2.0)$ \\
\hline Strongyloides stercoralis & 5 (1.3) & $2(0.3)$ \\
\hline Schistosoma haematobium & $0 \quad(0.0)$ & $6 \quad(0.9)$ \\
\hline Enterobius vermicularis & $3(0.8)$ & $0 \quad(0.0)$ \\
\hline
\end{tabular}

tle portion of their early morning feces. In the laboratory, each collected sample was examined using direct fecal smear and brine concentration methods. For the brine concentration method, harvesting of helminth ova and larvae was done using coverslips, which was left on the brine-fecal sample suspension for 3-5 min. All microscopic observations were done with 10x objective and, when required, 40x objective lenses. Egg counts were recorded as no. of eggs/g of feces. The chi-square $\left(\chi^{2}\right)$ test was used to compare data and to determine the significance of differences between prevalences.

Out of the 1,059 school children examined, 701 (66.2\%) had one or more helminth infection (s). The helminths identified and their prevalences were Ascaris lumbricoides (53.4\%; 565 cases), hookworms (17.8\%; 189 cases), Trichuris tichiura (10.4\%; 110 cases), Taenia sp $(9.6 \%$; 102 cases), Schistosoma mansoni (2.3\%; 24 cases), Strongyloides stercoralis ( $0.7 \% ; 7$ cases), Schistosoma haematobium ( $0.6 \% ; 6$ cases), and Enterobius vermicularis $(0.3 \% ; 3$ cases). The prevalence of A. lumbricoides was the highest, compared with other parasite species $(P<0.001)$. Table 1 compares the prevalences of the helminths among the urban center and the rural communities in the study area. The prevalence of T. trichiura in the rural communities was statistically higher than that in the urban community ( $P$ $<0.01$ ). For A. lumbricoides, hookworms, Taenia sp, $S$. mansoni and $S$. stercoralis, the prevalences among the
Table 2. Prevalence of parasitic helminths in relation to gender of the studied population in southwest Nigeria

\begin{tabular}{|c|c|c|}
\hline \multirow[t]{2}{*}{ Helminths } & \multicolumn{2}{|c|}{ No. (\%) infected } \\
\hline & Males & Females \\
\hline No. of cases examined & 524 & 535 \\
\hline Ascaris lumbricoides & $259(49.4)$ & $306(57.2)$ \\
\hline Hookworms & $90(17.2)$ & $99(18.5)$ \\
\hline Trichuris trichiura & $53(10.1)$ & $57(10.7)$ \\
\hline Taenia & $54(10.3)$ & $48 \quad(9.0)$ \\
\hline Schistosoma mansoni & $12(2.3)$ & 12 (2.3) \\
\hline Enterobius vermicularis & $2(0.4)$ & $1(0.2)$ \\
\hline Strongyloides stercoralis & $4 \quad(0.8)$ & $3(0.6)$ \\
\hline Schistosoma haematobium & $3(0.6)$ & $6 \quad(0.6)$ \\
\hline
\end{tabular}

urban center and rural communities were similar $(P>$ 0.05).

The gender-related prevalence of the helminths in the study area is shown in Table 2. Among males and females, A. lumbricoides revealed the highest prevalence $(P<0.001)$. Each of the recorded helminths had statistically similar prevalences among both genders $(P>0.05)$. The age-related prevalence of the helminths in the study area is summarized in Table 3. Ascaris lumbricoides showed the highest prevalence in all the age groups except $18+$ years $(P<0.05)$. The $18+$ years subjects had the statistically highest prevalence of $T$. trichiura $(P<0.001)$. For hookworms, $15-17$ years age group had statistically highest prevalence $(P<0.05)$. The remaining helminths recorded had similar prevalences among the infected age groups $(P>0.05)$.

Ascaris lumbricoides had fertile and infertile eggs ratio of 13: 1 and 3.7: 1 in the urban centre and rural areas, respectively. In the study area, $S$. haematobium had overall mean intensity of $650 \mathrm{eggs} / \mathrm{g}$. Among intestinal helminthes, A. lumbricoides had statistically highest mean intensity (565 eggs/g), followed by Taenia sp (475 eggs/g), hookworm (470 eggs/g), T. trichiura (460 eggs/g), S. stercoralis (190 larvae/g), E. vermicularis (150 eggs/g) and S. mansoni (145 eggs/g) $(P>0.05)$.

Among the infected school children, 244 (34.8\%), 29 (4.2\%), and $2(0.3 \%)$ had double, triple and quadruple infections, respectively. The prevalences of double infections among males (48.4\%) and females (51.6\%) 
Table 3. Prevalence of parasitic helminths in relation to age groups of studied population in southwest Nigeria

\begin{tabular}{|c|c|c|c|c|c|c|}
\hline \multirow{2}{*}{ Helminths } & \multicolumn{6}{|c|}{ No. $(\%)$ infected cases by age (yr) } \\
\hline & $3-5$ & $6-8$ & $9-11$ & $12-14$ & $15-17$ & $18+$ \\
\hline Ascaris lumbricoides & $6(60.0)$ & $82(60.7)$ & $240(56.1)$ & $156(51.3)$ & $79(46.2)$ & $2(18.1)$ \\
\hline Hookworms & $1(10.0)$ & $22(16.3)$ & $62(14.5)$ & $60(19.7)$ & $43(25.1)$ & $1 \quad(9.1)$ \\
\hline Trichuris trichiura & $0 \quad(0.0)$ & $9(6.7)$ & $41 \quad(9.6)$ & $23(7.6)$ & $33(19.3)$ & $4(36.4)$ \\
\hline Taenia sp. & $1(10.0)$ & $11(8.1)$ & $40 \quad(9.3)$ & 35 (11.5) & $13(7.6)$ & $2(18.1)$ \\
\hline Schistosoma mansoni & $0 \quad(0.0)$ & $5 \quad(3.7)$ & $6(1.4)$ & $8(2.6)$ & $5(2.9)$ & $0 \quad(0.0)$ \\
\hline Enterobius vermicularis & $0 \quad(0.0)$ & $0 \quad(0.0)$ & $2(0.5)$ & $1(0.3)$ & $0 \quad(0.0)$ & $0 \quad(0.0)$ \\
\hline Strongyloides stercoralis & $0 \quad(0.0)$ & $2(1.5)$ & $2(0.5)$ & $3(1.0)$ & $0 \quad(0.0)$ & $(0.0)$ \\
\hline Schistosoma haematobium & $0 \quad(0.0)$ & $2(1.5)$ & $4 \quad(0.9)$ & $0 \quad(0.0)$ & $0 \quad(0.0)$ & $0 \quad(0.0)$ \\
\hline
\end{tabular}

were statistically similar $(P>0.05)$. For double infections, Ascaris + hookworm combination had the statistically highest prevalence (45.9\%), followed by Ascaris + Trichuris (26.2\%), Ascaris + Taenia (16.0\%), hookworm + Trichuris (3.7\%), Ascaris + S. mansoni (2.9\%), hookworm + Taenia $(2.0 \%)$, hookworm + S. mansoni $(1.2 \%)$, Ascaris + Strongyloides $(0.8 \%)$, Trichuris + Taenia $(0.8 \%)$, and $S$. mansoni + Taenia $(0.4 \%)(P<0.001)$. The prevalences of triple infection among males $(37.9 \%)$ and females $(62.1 \%)$ were significantly different $(P<$ $0.02)$. The triple combination with the statistically highest prevalence was Ascaris + hookworm + Trichuris (48.3\%), followed by Ascaris + hookworm + Taenia $(17.2 \%)$, Ascaris + hookworm + S. mansoni $(13.8 \%)$, Trichuris + Taenia + S. mansoni $(10.3 \%)$, Ascaris + hookworm + Strongyloides (3.4\%), Ascaris + Taenia + Trichuris (3.4\%), and Ascaris + Taenia + S. mansoni (3.4\%) $(P<0.001)$. The two cases of quadruple infection were found among females and the only combination recorded was Taenia + S. mansoni + E. vermicularis + S. stercoralis.

Observations from questionnaires showed that $13.5 \%$ of the school children examined practised open and indiscriminate defaecation, while $34.8 \%$ indulged in walking bare-footed. Among those positive for $A$. lumbricoides $65.8 \%$ drank well and/or stream water, $46.1 \%$ nibbled finger nails, $40.8 \%$ failed to wash hands and/or fruits before eating and $80.3 \%$ claimed having been dewormed $\leq$ one year earlier.

The high prevalence of parasitic helminths (66.2\%) among school children in this study suggests a generally low standard of living and poor environmental sanitation in the study area (Ukoli, 1984; Smyth, 1996). The observation that $A$. lumbricoides had the highest prevalence in both the urban centre and rural communities agreed with the previous report of Ogbe et al. (2002) but disagreed with those of Nwaorgu et al. (1998) and Chhakda et al. (2006).The high prevalence of ascariasis among the children in the study area is corroborated by the relatively high occurrence of unhygienic habits among them. The similar prevalence of $A$. lumbricoides in the urban centre and rural communities possibly shows no difference among the communities in environmental sanitation and personal hygiene of the school children. The unhygienic practices among food vendors in the study area might have contributed to the occurrence of $A$. lumbricoides and some other helminthes among the school children (Idowu and Rowland, 2006). To the best of our knowledge, the report of the fertile and infertile eggs ratio of A. lumbricoides in this study is the first of its kind from Ogun State, southwest Nigeria. It is surprising that $A$. lumbricoides had higher proportion of fertile eggs in the urban centre than in the rural communities. This indicates the presence of relatively more viable transmissible A. lumbricoides eggs in the urban centre.

The prevalences of hookworm in the urban centre (17.9\%) and the rural communities (17.8\%) agreed with the report of Ogbe et al. (2002). However, the higher prevalence of $T$. trichiura in the rural communities compared to the urban centre is contrary to expectation in view of the fact that similar conditions generally enhance the endemicity of this infection, A. lumbricoides and hookworm (Ukoli, 1984; Piekarski, 1989). 
The reason for this discrepancy in the study area is not known. Taenia sp had similar prevalences in the urban centre and the rural communities. The presence of taeniasis in the study area might have been due partly to widespread habit of eating improperlycooked beef (locally known as 'suya') and pork in the study area. Taeniasis had been reported previously from some other parts of Nigeria (Gundiri and Okwuosa, 2005) and elsewhere (Celiksoz et al., 2005). The prevalences of E. vermicularis and S. stercoralis were low in the study area. This might have been due to the diagnostic methods used in this study; particularly for E. vermicularis which is best diagnosed using cellophane tape smear methods (Shoup, 2001). The occurrence of S. mansoni in the study area conforms to some previous reports from Nigeria (Bassey and Umar 2004). The observation of S. haematobium eggs in feces of some school children in the study area agrees with the fact that they sometimes appear in the feces of infected persons (Smyth, 1996). The presence of schistosomiasis is no surprise since many of the school children had cause to frequent streams for drinking water, laundry, and other domestic purposes. Collection of edible freshwater snails (particularly Lanistes libycus) had been noted an important reason for water contact by many school children in many parts of southwestern Nigeria (Agbolade et al., 2004).

The study showed that although A. lumbricoides was most prevalent in both genders, none of the parasitic helminthes was gender-dependent in conformity with some previous reports (Mafiana and Omotayo, 1998; Taiwo and Agbolade, 2000). Generally, ascariasis decreased gradually in prevalence as the age of the school children increased. However, the high prevalence in virtually all age groups indicates general ignorance and/or deliberate neglect of basic rules of hygiene among the children. The observation of relatively higher prevalences of hookworm in age groups 12-14 and 15-17 years may be an indication of their higher level of involvement in farming activities than others. The relatively high prevalence of Taenia sp in children of 18 years age may be because they feel free in their communities and have more to spend on 'suya'. However, the reason for 18 years old children having highest prevalence of T. trichiura is not known. The absence of $S$. mansoni among 3-5 years age group may be because they make infrequent (if any) contact with natural water bodies. Normally, most of the eggs of S. haematobium are found in urine samples (Smyth, 1996). Therefore, a definite conclusion on the agerelated occurrence of $S$. haematobium is difficult since no microscopic examination of urine samples was done in this study. Nevertheless, a study involving testing of urine samples with diagnostic reagent strips in the area will be reported in a later paper. The seeming restriction of $S$. stercoralis and E. vermicularis to certain age groups is attributable to their low prevalences in this study. The intensity of the helminthes appeared generally low in the study area.

In this study, the most common double infection was Ascaris + hookworm, while the most common triple infection was Ascaris + hookworm + Trichuris. These possibly indicate that the environmental conditions in the study area satisfied the development and successful transmission of A. lumbricoides, hookworm, and T. trichiura simultaneously (Ukoli, 1984).

This study has shown a general similarity in the epidemiological pictures of parasitic helminthes in the urban centre and the rural communities in the study area. This corroborates the opinion of Gillespie (2001) that urbanisation and population growth often combine to increase total burden of diseases in developing countries. It necessarily implies that there is an urgent need for concerted efforts towards ensuring adequate control of intestinal helminthiasis and schistosomiasis in OLGA and ILGA of Ogun State, Nigeria.

\section{ACKNOWLEDGMENTS}

We sincerely thank the local education authorities of Odogbolu and Ijebu-Ode Local Government areas of Ogun State, southwest Nigeria, for their permission to conduct this survey. We also appreciate the cooperation of the heads, teachers, parents, and children of the schools visited during the present study. 


\section{REFERENCES}

Agbolade OM, Akinboye DO, Fajebe OT, Abolade OM, Adebambo AA (2004) Human urinary schistosomiasis transmission foci and period in an endemic town of Ijebu North, Southwest Nigeria. Trop Biomed 21: 15-22.

Ahmed SG, Ibrahim UA, Ekemu ED (2004) Prevalence of intestinal parasites in anaemic and non-anaemic Nigerian patients with acquired immune deficiency syndrome. Nigerian J Parasitol 25: 15-20.

Bassey SE, Umar Z (2004) A re-assessment of schistosomiasis infection in Garun-Babba, Kadawa and Kura in Kano State, Nigeria. Nigerian J Parasitol 25: 107-109.

Anosike JC, Nwoke BE, Njoku AJ (2001) The validity of haematuria in the community diagnosis of urinary schistosomiasis infection. J Helminthol 75: 223-225.

Chhakda T, Muth S, Socheat D, Odermatt P (2006) Intestinal parasites in school-aged children in villages bordering Tonle Sap Lake, Cambodia. Southeast Asian J Trop Med Public Health 37: 859-864.

Clennon JA, King CH, Muchiri EM, Kariuki HC, Ouma JH, Mungai P, Kitron U (2004) Spatial pattern of urinary schistosomiasis infection in a highly endemic area of coastal Kenya. Am J Trop Med Hyg 70: 443-448.

Ekpo UF, Mafiana CF (2004) Epidemiological studies of urinary schistosomiasis in Ogun State, Nigeria: Identification of high-risk communities. Nigerian J Parasitol 25: 111-119.

Gillespie SH (2001) Intestinal nematodes. In Principles and Practice of Clinical Parasitology, Gillespie SH, Pearson RD (eds.). p 561-583, John Wiley \& Sons Ltd., Chichester, England.

Gundiri MA, Okwuosa VN (2005) Prevalence of urinary and intestinal parasites in Kwampe, Langtang North, Nigeria. Nigerian J Parasitol 26: 19-22.

Idowu OA, Rowland SA (2006) Oral fecal parasites and personal hygiene of food handlers in Abeokuta, Nigeria. Afr Health Sci 6: 160-164.

Mafiana CF, Omotayo AB (1998) Urinary schistosomiasis:
An evaluation of microscopic egg count and chemical reagent strip in children in south-west Nigeria. Helminthologia 35: 31-36.

Nokes C, Cooper ES, Robinson BA, Bundy DA (1991) Goehelminth infection and academic assessment in Jamaican children. Trans $R$ Soc Trop Med Hyg 85: 272273.

Nwaorgu OC, Okeibunor J, Madu E, Amazigo U, Onyegegbu N, Evans D (1998) A school-based schistosomiasis and intestinal helminthiasis control programme in Nigeria: acceptability to community members. Trop Med Int Health 3: 842-849.

Ogbe MG, Edet EE, Isichei MN (2002) Intestinal helminth infection in primary school children in areas of operation of Shell Development Company of Nigeria (SPDC), western division in Delta State. Nigerain J Parasitol 23: 310.

Poggensee G, Krantz I, Nordin P, Mtweve S, Ahlberg B, Mosha G, Freudenthal S (2005) A six-year follow-up of schoolchildren for urinary and intestinal schistosomiasis and soil-transmitted helminthiasis in Northern Tanzania. Acta Trop 93: 131-140.

Shoup B (2001) Diagnosis and management of pinworm infection. Prim Care Update Ob Gyns 8: 240-243.

Smyth JD (1996) Animal Parasitology. pp 236-246, 397-422, Cambridge University Press, Cambridge, UK.

Taiwo AK, Agbolade OM (2000) Intestinal helminthiasis among school children in Oru, Ogun State, Nigeria. Nigerian J Sci 34: 283-286.

Ukoli FMA (1984) Introduction to parasitology in tropical Africa. pp 227-267, John Wiley \& Sons Ltd., Chichester, UK.

Utzinger J, Vounatsou P, N'Goran EK, Tanner M, Booth M (2002) Reduction in the prevalence and intensity of hookworm infections after praziquantel treatment for schistosomiasis infection. Int J Parasitol 32: 759-765.

WHO (1993) The control of schistosomiasis: second report of the WHO Expert Committee. p1-4, World Health Organization, Geneva, Switzerland. 